Supporting Information for Publication

\title{
Synergy in the Cracking of a Blend of Bio-oil and Vacuum Gasoil under Fluid Catalytic Cracking Conditions
}

Álvaro Ibarra $^{l}$, Elena Rodríguez ${ }^{l}$, Ulises Sedran ${ }^{2}, J_{o s e ́}$ M. Arandes $^{l}$, Javier Bilbao $^{l}$

${ }^{1}$ Chemical Engineering Department, University of the Basque Country (UPV/EHU), P. O. Box 644, 48080. Bilbao, Spain.* Telephone: +3494601-2511, Email: alvaro.ibarra@ehu.es

${ }^{2}$ Instituto de Investigaciones en Catálisis y Petroquímica INCAPE (FIQ, UNL-CONICET), Santa Fe, Argentina. 
Table S1. Composition of the raw bio-oil.

\begin{tabular}{lc}
\hline Elemental composition (wt $\%)$ & \\
\hline $\mathrm{C}$ & 55.1 \\
$\mathrm{H}$ & 6.3 \\
$\mathrm{~S}$ & - \\
$\mathrm{O}$ & 38.6 \\
\hline Water content (wt $\%)$ & 46.5 \\
\hline Composition (wt $\%)$ & \\
\hline Acids and esters & 21.6 \\
Acetic acid & 15.5 \\
Aldehydes & 19.4 \\
Hydroxy-acetaldehyde & 7.8 \\
Ketones & 16.3 \\
$1-$ Hydroxy-2-propanone & 6.5 \\
Phenols & 5.1 \\
Alcohols & 9.7 \\
Ethers & 2.8 \\
Sugars & 24.1 \\
Levoglucosan & 21.6 \\
Non identified & 1.0 \\
\hline Simulated Distillation $\left({ }^{\circ} \mathrm{C}\right), \mathrm{D}-2887$ & 35 \\
Initial boiling point & 55 \\
5 wt $\%$ & 74 \\
$25 \mathrm{wt} \%$ & 154 \\
50 wt $\%$ & 263 \\
$90 \mathrm{wt} \%$ & 335 \\
Final boiling point &
\end{tabular}


Table S2. Composition of the vacuum gasoil (VGO).

\begin{tabular}{lc}
\hline Density $\left(\mathrm{g} \mathrm{cm}^{-3}\right)$ & 0.918 \\
Average molecular weight $\left(\mathrm{g} \mathrm{mol}^{-1}\right)$ & 370.3 \\
\hline Elemental composition $(\mathrm{wt} \%)$ & \\
\hline $\mathrm{C}$ & 85.4 \\
$\mathrm{H}$ & 11.8 \\
$\mathrm{~S}$ & 2.5 \\
$\mathrm{O}$ & - \\
\hline Composition (wt \%) & \\
\hline Aliphatics & 47.2 \\
Aromatics & 43.1 \\
Sulfur compounds & 9.7 \\
\hline Simulated Distillation $\left({ }^{\circ} \mathrm{C}\right), \mathrm{D}-2887$ & 184 \\
\hline Initial boiling point & 367 \\
5 wt \% & 432 \\
25 wt \% & 474 \\
50 wt \% & 533 \\
90 wt \% & 614 \\
Final boiling point &
\end{tabular}

